\title{
An Hybrid Wigner-Ville Approach to Estimate the Ultrasound Attenuation in Soft Biological Tissue
}

\author{
Chemla J.P., Girault J.M., Student Member IEEE, Kouamé D. and Lethiecq M., Member, IEEE.
}

\begin{abstract}
In this paper, we compare several techniques to evaluate the frequency shift related to the attenuation phenomenon. More precisely, we compare the short time Fourier analysis and the short time autoregressive analysis to new techniques based upon the Wigner-Ville transform with paranctric and non parametric approacles. In the parametric metlods, we propose a new frequency estimator called the mean resonating frequency. All of these methods are evaluated in terms of relative error and standard deviation of the attenuation estimation.
\end{abstract}

ficyuords - Ultrasound, Tissue Characterization, Attenuation estimation, time-frequency analysis, parametric, nonparametric.

\section{INTROUUCTION}

$\mathrm{I}$ T $N$ the field of tissue characterization, ultrasound attenLuation has proved to be a discriminant quantitative parameter which is complementary to the qualitative information obtained from echographic imnges. The attenuation phenomenon shifts the instautaneous spectrum to lower frequencies as a pulse wave propagates into the biological tissue. The spectral analysis of nonstationary signals requires a signal processing tool that presents both good time and frequency resolutions. It is well-known that the most commonly used short-time Fourier transform is limited by the intrinsic trade-of between time and frequency resolutions.

To overcome this resolution problem three approaches are possible: (i) the Wigner-Ville transform which allows a better compromise, (ii) the parametric methods and (iii) an hybrid method which is based on the Wigner-Ville trans. form (non parametric) computed by a parametric technique.

lit the paranetric methods, we propose a new frequency sstimator: the minu resoluating freguency. It is compared to the centroid and the maximum energy frequencies [1].

In this paper, we quantify the performances of these methods compared to those of the short time Fourier analysis.

\section{ATtenuation modeling and simulated data}

In order to compare the proposed methods, we have built artificial signals that simulate the Radio-Frequency (RF) lines reflected by a soft biological tissue. This modeling

\footnotetext{
Chemla J.P. and Lethiecq M. are with the LUSSI/EA2102, EIVL, rue de la chocolaterie BP 3410, 41034 Blois Cedex, France. Girault J.A. and KuUame D. are with LUSSI/EA2102, GIP Ultrasons, 37032 Tours Cedex, France. E-mail: chemlaguniv-tours.fr

This work was spunsorised by the Region Centre of France.
}

takes into account the pulse response of the measurement system, the absorption and the scattering of the medium. The diffraction phenomenon which biases the attenuation measurement could be compensated by a filter [2], so we did not consider it in our nodel [3]. It is usually accepted that attenuation $\alpha$, in most biological tissues, is a linear function of the frequency: $\alpha=\beta f$ where $\beta$ is the slope of the attenuation.

It is also considered that the spectral density of the emitted signal has a gaussian form.

The simulated echographic lines are obtained with a sampling frequency of $400 \mathrm{MHz}$, an emitted central frequency of $45 \mathrm{M} \mathrm{Hz}$ and a bandwidth of $50 \%$. The homogeneous medium is simulated for different attenuation coefficient values ranging from 1 to $5 \mathrm{~dB} / \mathrm{cm} / \mathrm{MHz}$. For each attenuation coefficient value, a set of 100 RF lines are conputed. With a wave velocity of $3530 \mathrm{~m} / \mathrm{s}$, the duration of the backscattered signal corresponds to a depth of $2 \mathrm{~mm}$.

\section{THE TIME-FREQUENCY ANALYSIS}

The attenuation phenomenon is usually observed in the frequency domain through the frequency shift observed as the ultrasound pulse propagates in the soft medium. This time-frequency analysis is generally performed by a Fast Fourier Transform (FFT) [4].

\section{A. The short time Fouricr analysis}

Owing to the nonstationary character of the radiofrequency signal, a Hamming window is shifted with a constant step along the signal and in each window, the Power Spectral Density (PSD) is evaluated via the computation of the FF'l', 'l'his method implies that, we have to thake a thoice for the length and the overlap of the windows. Indeed, the larger the windows, the better is the frequency resolution but the less the stationarity hypothesis is verified. The choice is obtained by a trade-off between the frequency and time resolutions. In order to have a better compromise, we propose the use of the Wigner-Ville transform.

\section{B. The Wigner-Ville (WV) transform}

The Wigner-Ville distribution is a well adapted tool to study nonstationary phenomena. It seems to have a good behavior for reflected signals [3].

The Wigner-Ville distribution is defined by :

$$
W(t, f)=\int_{-\infty}^{\infty} \zeta(t+\tau / 2) \zeta^{\star}(t-\tau / 2) c^{-2 \pi j f \tau} d \tau
$$


where, in our sludy, $\zeta(t)$ is the analytical signal of the RF signal and $\zeta(t+\tau / 2) \zeta^{\star}(t-\tau / 2)$ is the kernel. We used the muncrical implementation of the windowed discrete Wiguer-Vil] distribution [5]. This distribution is defined by :

$H^{g}(n, f)=\sum_{m=-(M-1) / 2}^{m=(M-1) / 2} g(m) \zeta(n+m) \zeta^{*}(n-m) e^{-4 \pi j J m / M}$

where $g(m)$ is the Hanming window. Another solution is to analyze a modeled signal : this is, for example, the case in autoregressive modeling.

\section{Autoregressive (AR) modeling}

The echographic signal is modeled as the output of linear filter driven by a complex white Gaussian noise $u(n)$ with zero mean variance $\sigma_{u}^{2}[6]$ :

$$
\zeta(n)=-\sum_{i=1}^{p} a_{i} \zeta(n-i)+u(n)
$$

where $a_{i}$ are the complex autoregressive parameters and $p$ is the order of the AR model. $\zeta(n)$ is the analytical windowed signal. The optimal AR order is obtained (see figure 1) when both the standard deviation and relative error of the attenuation coefficient are the lowest. There are many algorithms to compute AR parameters required for the poles and spectrum estimation. We will focus on the most popular onc: the least-square algorithm [6], [1]. linally, from known An paraneters the whole spectrum can be evaluated.

\section{The autoregressive Wigner-Ville $(A R V W)$ method}

This method is a mix between the Wigner-Ville method and the autoregressive method. In order to compute the Fourier transform of the kernel as in the conventional Wigner-Ville approach, we inplement the computation of the square-root of the AR kernel spectrum. This is not restrictive, others solutions can be proposed.

\section{Frequency estimators}

\section{A. Parametric frequency estimators}

The whole spectrum can be cvaluated using the AR parrameters and the corresponding poles:

$$
\begin{gathered}
H(z)=\frac{1}{1+\sum_{i=1}^{p} a_{i} z^{-i}} \\
S(f)=\sigma_{u}^{2}|H(z)|_{z=\exp (-2 \pi j f)}^{2} \\
S(f)=\frac{\sigma_{u t}^{2}}{\left|\prod_{i=1}^{p}\left(z-z_{i}\right)\right|_{z=\exp (-2 \pi j f)}^{2}}
\end{gathered}
$$

where $f$ is the normalized frequency $0 \leq f \leq 0.5$ and $\sigma_{u}^{2}$ is the white noise energy. The centroid frequency [8] and the maximum energy frequency [7] can be evaluated directly on the spectrum. The centroid is given by :

$$
f_{c}=\frac{\int_{-\infty}^{+\infty} f S(f) d f}{\int_{-\infty}^{+\infty} S(f) d f}
$$
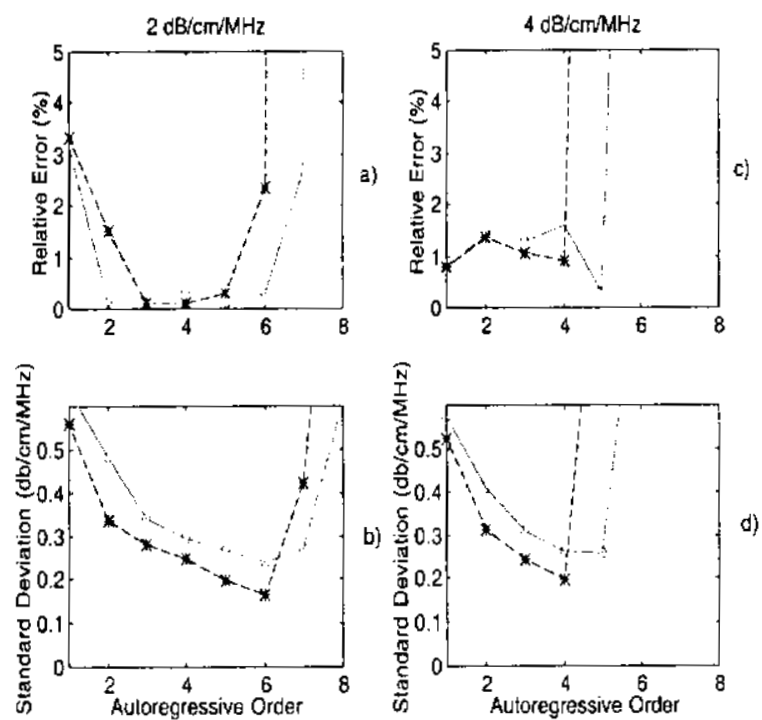

Fig. 1.

Relative Error in \% (a and c) and Standard Deviation in $\mathrm{dB} / \mathrm{cm} / \mathrm{MHz}(\mathrm{b}$ and $d)$ of the altenuation coefficient for 2 and $4 \mathrm{~dB} / \mathrm{cm} / \mathrm{MHz}$ versus the Autoregressive Order computed via the mean resonating frequency. Dark plot (with*) corresponds to the autoregressive method; Light plot (with o) corresponds to the aturergressier Wiyner-Villc method.

The resonating freguencies (see Figure 2) that correspond to the pole $p_{i}$ are evaluated by:

$$
f p_{i}=f e \frac{\arg \left(\check{z}_{i}\right)}{2 \pi}
$$

with $\arg \left(z_{i}\right) \in[0, \pi]$ and where $f e$ is the sampling frequency. In this case the mean resonating frequency is given by:

$$
f_{n r}=\frac{f e}{p 2 \pi} \sum_{i=1}^{p} \arg \left(z_{i}\right)
$$

\section{B. Non parametric frequency cstimators}

In the non parametric case, only the centroid frequency is authorized. Indeed, as variance of the frequency estimation evaluated by the maximum energy frequency is too high (due to the randon character of the spectrum), it can not be used.

Figure 3 illustrates the evolution of $f_{c}$ in the nonparametric case and $f_{m r}$ in parametric case. These curves are averaged with $100 \mathrm{RF}$ lines. These curves are almost parallel to those of theoretical frequencies. A significant bias appears for non-paranetric approaches, but it does not dislurb atienuation estimation because its slope constitutes the relevant information. 


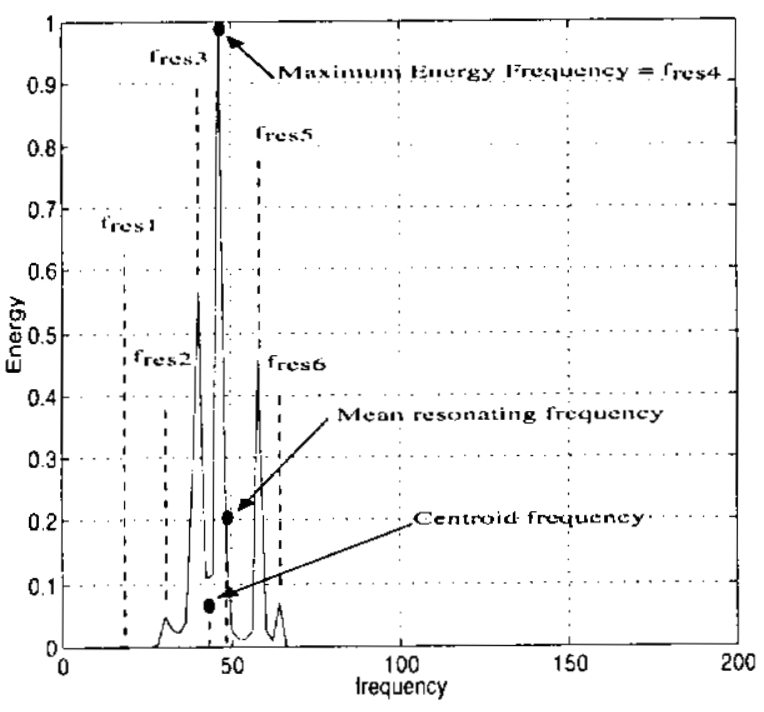

Fig. 2.

Autoregressive spectrum wilh a 6 order $A R$ model.

\section{RESULTS}

First, we compare the presented parametric methods in terms of relative error and standard deviation of the attenuation estimate (see Table I). This is performed for a theoretical attenuation coefficient of $3 \mathrm{~dB} / \mathrm{cm} / \mathrm{MHz}$, with 100 simulated RF lines, by means of the three presented frequency estimators. These results are obtained with a 4 order autoregressive model. Indeed, Figure l indicates that a 4 order autoregressive model is well adapted to our application. Similar results are obtained with other attenuation coefficients (from 1 to $5 \mathrm{~dB} / \mathrm{cm} / \mathrm{MHz}$ ).

TABLE I

\begin{tabular}{|l|c|c|c|c|}
\hline \multirow{2}{*}{$\begin{array}{l}\text { Frequency } \\
\text { stimators }\end{array}$} & \multicolumn{4}{|c|}{ Methods } \\
\cline { 2 - 5 } & Autoregressive & \multicolumn{2}{|c|}{ AR Wigner-Ville } \\
\cline { 2 - 5 } & 13. & SD & IRE & SD \\
\hline \hline Controid & 0.56 & 0.55 & 0.3 & 0.15 \\
Maximum Energy & $\leq 0.1$ & 0.91 & 3.0 & 0.82 \\
Mean resonating & 0.8 & 0.22 & 0.1 & 0.30 \\
\hline
\end{tabular}

Relative Error ( $R E$ in \%) and Standard Deviation (SD in $\mathrm{d} B / \mathrm{cm} / \mathrm{MHz}$ ) for a theoretical attenuation cocfficient of $3 \mathrm{~dB} / \mathrm{cm} / \mathrm{MHz}$ with a 4 order autoregressive method.

Thus, table I points out that in the case of the classical centroid and maximum energy frequencies, the best method, in term of standard deviation, is the bybrid Wigner-Ville. This is due to the combination of properties of the conventional Wigner-Ville and parametric methods.

Let us now consider the new frequency estimator : the mean resonating frequency. Table I highlights that the best attenuation estimation is given by the autoregressive

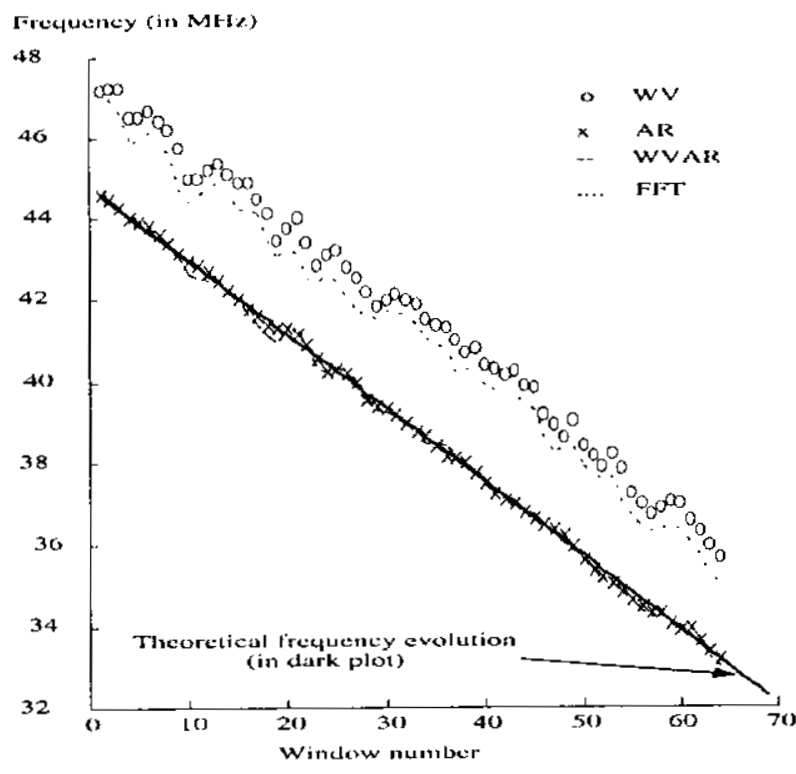

Fig. 3.

The time freguency tracking wilh the four mothods for a theoretical allemation cosficion of $3 \mathrm{~dB} / \mathrm{cm} / \mathrm{MHz}$.

method via the computation of the mean resonating frequency. We can stipulate that this good behavior is due to the fact that we have performed both stalistical (100 RF lines) and frequency averages (corresponding to the pole arguments). These results suggest the joint use of mean resonating frequency cstimator and autoregressive modeling.

The results of a comparison between parametric and non parametric methods is summarized in Tables II and III.

TABLE II

\begin{tabular}{|c|c|c|c|c|}
\hline \multirow{3}{*}{$\begin{array}{c}\text { Theoretical } \\
\text { atlomuation } \\
\text { in } \mathrm{d} \mathrm{H} / \mathrm{cm} / \mathrm{M} / \mathrm{z}\end{array}$} & \multicolumn{4}{|c|}{ Methods } \\
\hline & \multicolumn{2}{|c|}{ Non Parametric } & \multicolumn{2}{|c|}{ Parametric } \\
\hline & Fl] & WV & $\Lambda R$ & $\Lambda R W V$ \\
\hline 1 & 1.20 & 3.60 & 3.10 & 4.40 \\
\hline 2 & 2.45 & 0.90 & 0.10 & 0.35 \\
\hline 3 & 0.17 & 0.53 & 0.87 & 0.10 \\
\hline 4 & 0.30 & 0.75 & 0.93 & 1.58 \\
\hline 5 & 0.32 & 0.44 & 0.42 & 1.10 \\
\hline
\end{tabular}

Relative Error (in \%) of the atlcnuation coefficient estimate in the case of parametric and non-parametric methods

The non-parametric Wigner-Ville approach improves the standard deviation of the attenuation estimate, as compared to the Fourier approach, by a factor of $14 \%$. This factor increases to around $45 \%$ for the hybrid Wigner-Ville approach and to around $58 \%$ for the AR approach. 
TABLE III

\begin{tabular}{|c|c|c|c|c|}
\hline \multirow{3}{*}{$\begin{array}{l}\text { Theoredical } \\
\text { attenuation } \\
\text { in } \mathrm{d} / \mathrm{B} / \mathrm{m} / \mathrm{H} / \mathrm{H}=\end{array}$} & \multicolumn{4}{|c|}{ Methorls } \\
\hline & \multicolumn{2}{|c|}{ Non Parinutric } & \multicolumn{2}{|c|}{ Parametric } \\
\hline & {$\left[+I^{2}\right.$} & $1 V^{\prime}$ & $A R$ & $\Lambda 1 \mathrm{WV}$ \\
\hline 1 & 0.543 & 0.479 & $0.226^{\circ}$ & 0.296 \\
\hline 2 & $0.5 \times 10$ & 0.180 & 0.219 & 0.205 \\
\hline 3 & 0.5417 & 0.457 & 0.222 & 0.302 \\
\hline 4 & 0.512 & 0.445 & 0.199 & 0.263 \\
\hline 5 & 0.521 & 0.428 & 0.231 & 0.301 \\
\hline
\end{tabular}

Stundard Deviation (in $d B / \mathrm{cm} / \mathrm{MHz}$ ) of the attenuation coefficicnt estimate in the case of parametric and non-parametric methods

\section{Conclusions}

In this paper, we have compared the performances of time-frequency methods such as the Fourier, the WignerVille and the autoregressive approaches as well as a new autoregressive Wigner-Ville method for attenuation coeflicient estimation. We have also presented a new way to estimate the frequency of parametric power spectral density and compared it with classical frequency estimators. These comparisons were performed with simulated R.F lines in biological fissues with different attemution cocllieient values ranging from 1 to $5 \mathrm{~dB} / \mathrm{cm} / \mathrm{MHz}$.

In the field of parametric analysis, we suggest the joint use of autoregressive models and the mean resonating frequency estimator. For non parametric analysis, the Wigner-Ville distribution leads to a better attenuation estimation. Indeed, the standard deviation of these methods is lower. Consequently the estimation of the attenuation coefficient should require a lower number of RF lines than for classical methods.

Our future work will be focuscd on the adaptation of the autoregressive order for each attenuation value as well as on the application of these methods to real echographic lines.

\section{REFERENCES}

[1] J.M. Girault, F. Ossant, A. Ouahabi, D. Kouame, and F. Patat, "Time-varying autoregressive spectral estimation for ultrasound attemuation in (issuc characterization," IEEE Trans UFFC, vol. 45. $p p .650 \cdot(659,1: 298$.

[2] M. Fink and J.l: Carcloso, "Diflraction effects in pulse echo measurement." IEEE Trans Sonic and Vltras, vol. 31, pp. 313-329, 1984.

[3] J.P. Chemla, J.M. Girault, D. Kouame, F. Ossant, and M. Lethiecq, "Use of the wigner-ville transform for ultrasound attenuation of biological tissues," Innov. Techn. Biol. Med, vol. 19 , pp. 213-219, 1998

[4] M. Fink, F. Hottier, and J.F. Cardoso, "Signal processing for in vivo attenuation measurement: Short time fourier analysis," Ultrasonic Imaging, vol. 5, pp. 117-135, 1983.

[5] B. Bouashash, Signal Analysis Processing, Advances in Spectrum Analysis and Array 'Time-Frequency Simon Haykin Editor Prentice Hall New Jersey, 1991.

[6] S.M. Kay and S.L. Marple, "Spectrum analysis - a modern perspective," Proc IEEE, vol. 69, pp. 1380-1419, 1981.

[i] T. Baldeweck, P. Laugier, A. Herment, and G. Berger, "Application of autoregressive spectral analysis for ultrasound at tenuation estimation: Interest in highly attenuation medium," IEEE Trans $U F F C$, vol. 42, pp. 99-110, 1995.

[8] R. Kuc and H. Li, "Reduced-order autoregressive modeling for center frecuency estimation," Ultrasonic Imatging, vol, 00, pl3. $2414-251,1985$

[9] G. Berger, P. Laugier, M. Fink, and J. Perrin, "Optimal precision in ultrasound at tenuation estimation and applicalion to the

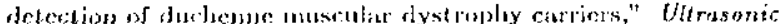
Imaging, wel. 9, pp. 1 17, 1:\$8 